\title{
Initial NIST AC QHR Measurements
}

\begin{tabular}{|c|c|}
\hline Volume 109 & Number 4 \\
\hline $\begin{array}{l}\text { M. E. Cage, S. H. Shields, and } \\
\text { A. Jeffery }\end{array}$ & $\begin{array}{l}\text { We demonstrate that dc quantized Hall } \\
\text { resistance (dc QHR) guideline properties } \\
\text { and dc and ac QHR values can be }\end{array}$ \\
\hline $\begin{array}{l}\text { National Institute of Standards } \\
\text { and Technology, } \\
\text { Gaithersburg, MD 20899-8172 }\end{array}$ & $\begin{array}{l}\text { measured without changing sample probe } \\
\text { lead connections at the QHR device, } \\
\text { and report ac QHR values that converge } \\
\text { to the dc QHR value when using } \\
\text { four-terminal-pair ac QHR measurements. }\end{array}$ \\
\hline $\begin{array}{l}\text { marvin.cage@nist.gov } \\
\text { scott.shields@nist.gov }\end{array}$ & $\begin{array}{l}\text { This was accomplished during one } \\
\text { cooldown using single-series and } \\
\text { quadruple-series connections outside the } \\
\text { sample probe. The QHR was measured } \\
\text { from } 0 \mathrm{~Hz} \text { to } 5500 \mathrm{~Hz} \text { in } 1: 1 \text { ratio at } \\
20 \mu \mathrm{A} \text { to } \pm 1 \text { part in } 10^{7} \text { uncertainties with } \\
\text { a poor-quality QHR device. A good } \\
\text { device would allow an order of magnitude } \\
\text { smaller uncertainties over this frequency } \\
\text { range. We exchanged positions of the } \\
\text { QHR device and reference resistor in the } \\
\text { bridge and remeasured the resistance ratios } \\
\text { to remove dominant ac bridge effects. }\end{array}$ \\
\hline
\end{tabular}

July-August 2004

\section{E. Cage, S. H. Shields, and effery and Technology, Gaithersburg, MD 20899-8172}

marvin.cage@nist.gov scott.shields@nist.gov

\author{
We demonstrate that de quantized Hall \\ 政 \\ lead connections at the QHR device, \\ to the $\mathrm{dc}$ QHR value when using \\ This was accomplished during one \\ cooldown using single-series and \\ sample probe. The QHR was measured \\ from $0 \mathrm{~Hz}$ to $5500 \mathrm{~Hz}$ in $1: 1$ ratio at \\ $20 \mu \mathrm{A}$ to \pm 1 part in $10^{7}$ uncertainties with \\ a poor-quality QHR device. A good \\ smaller uncertainties over this frequency \\ QHR device and reference resistor in the \\ to remove dominant ac bridge effects.
}

\begin{abstract}
Keywords: ac quantum Hall effect; dc quantum Hall effect; frequency dependences; multifrequency bridge; quadruple-series connections; single-series connections.
\end{abstract}

Accepted: September 5, 2004

Available online: http://www.nist.gov/jres

\section{Introduction}

An important step in developing the ac quantized Hall resistance (ac QHR) [1-10] as an intrinsic impedance standard based on the dc QHR [11-13] is to measure the dc QHR guideline properties [14] and the dc and ac QHR values without changing sample probe lead contacts at the QHR device. (Otherwise guideline properties should be remeasured.) We show in the first ac QHR experiment at NIST that this can be accomplished in a single cooldown using multiple connections [15] to the device outside the sample probe. The device was found to not be of standards-quality; so only guideline properties needed for this particular experiment were measured (shapes of the QHR plateaus and longitudinal voltages $V_{x}$, contact resistances, longitudinal resistances at the $V_{x}$ minima, dc QHR values for all three quantum Hall probes sets at the $V_{x}$ minima, and the quadruple-series-connected dc QHR value at the $V_{x}$ minimum). All dc guideline properties could have been determined for a good device (including those for magnetic field reversal) using the external single-series and quadruple-series configurations. (Of course the do guidelines are a necessary, but not sufficient, condition of ac device suitability, and any future proposed ac guidelines are yet another matter.)

The ac QHR values converged to the dc QHR value under proper conditions (which were external quadruple-series connections [16], four-terminal-pair (4TP) techniques $[17,18]$, and interchanged 1:1 ratio measurements). We wanted to demonstrate convergence to about \pm 1 part in $10^{8}$ of the dc QHR, but poor device properties limited the present uncertainty to \pm 1 part in $10^{7}$. 


\section{QHR Device, Header, and Sample Probe}

The only ac QHR device available was a LEP 175 GaAs/AlGaAs heterostructure fabricated by the Laboratories d'Electronic Philips ${ }^{1}$ in France that we labeled ac1. K. C. Lee of NIST mounted it on one of our custom-built headers using $100 \mu \mathrm{m}$ diameter platinum wires to avoid vibrational effects of ac currents in a magnetic field. The header, constructed from gold-patterned $1.6 \mathrm{~mm}(1 / 16 ")$ thick printed circuit board, had a single ground plane over most of its back surface to minimize wire-to-wire capacitances at the device. (This matters because Eq. (55) of Ref. [16] predicts that the largest frequency-dependent correction term in quadruply-connected ac QHR measurements is $-\omega^{2} C_{\mathrm{X}^{\prime} \mathrm{X}^{\prime}} C_{\mathrm{X}^{\prime} \mathrm{X}^{\prime}} R_{\mathrm{H}} R_{\mathrm{H}}$, which involves the squares of: the angular frequency $\omega$; the summed-total $C_{\mathrm{X}^{\prime} \mathrm{X}^{\prime}}$ of those wire-to-wire capacitances that have the quantum Hall voltage across them; and the quantized Hall resistance $R_{\mathrm{H}}$.) A single ground plane assures symmetry on magnetic field reversal. Tests showed that this grounded back-plane reduced wire-to-wire capacitances, with only slight increases in capacitances-to-shield, whereas a ground plane on the front surface of the header significantly increased capacitances-to-shield for similar reductions in wire-to-wire capacitance.

We predipped the eight semi-rigid coaxial cables several times in liquid helium before sample probe assembly to minimize Teflon insulation flow during cooldown. (B. W. Ricketts of the National Measurement Laboratory in Australia cautioned us that differential compression when cooling the coaxial cables squeezes the Teflon, causing it to flow during the first few cooldowns and thus stress solder joints at the coaxial socket.)

Cernox thermometers sensitive to a $\mathrm{mK}$ were located above and below the device, which could only be cooled to $1.5 \mathrm{~K}$ at maximum pumping rates (rather than the $1.3 \mathrm{~K}$ of our dc sample probes) because semi-rigid coaxial cables have more thermal conduction.

\section{Device Properties}

We pumped the sample probe extensively to avoid trapped gasses in the coaxial leads, and then cooled the

\footnotetext{
${ }^{1}$ Certain commercial equipment, instruments, or materials are identified in this paper to foster understanding. Such identification does not imply recommendation or endorsement by the National Institute of Standards and Technology, nor does it imply that the materials or equipment identified are necessarily the best available for this purpose.
}

device slowly over two days to eliminate electron-hole pair production in the device (which creates additional electrons in the two-dimensional fluid and a slow downward drift in the magnetic field positions of QHR plateaus as the pairs recombine). The device was then maintained at or below $4.2 \mathrm{~K}$ for 3 months.

Figure 1 shows a magnetic field sweep for the central quantum Hall voltage probes $V_{\mathrm{H}}(3,4)$ and the longitudinal voltage probes $V_{x}(2,6)$ at a $I=20.0 \mu \mathrm{A} \mathrm{dc}$ source-drain current and $T=1.59 \mathrm{~K}$ temperature. (The device source, drain, and potential probe contacts $\mathrm{S}^{\prime}$, $\mathrm{D}^{\prime}, 1^{\prime}-6^{\prime}$ are identified in Fig. 7. Primes refer to connections at the device contact pads, while unprimed numbers and letters refer to connections outside the sample probe.)

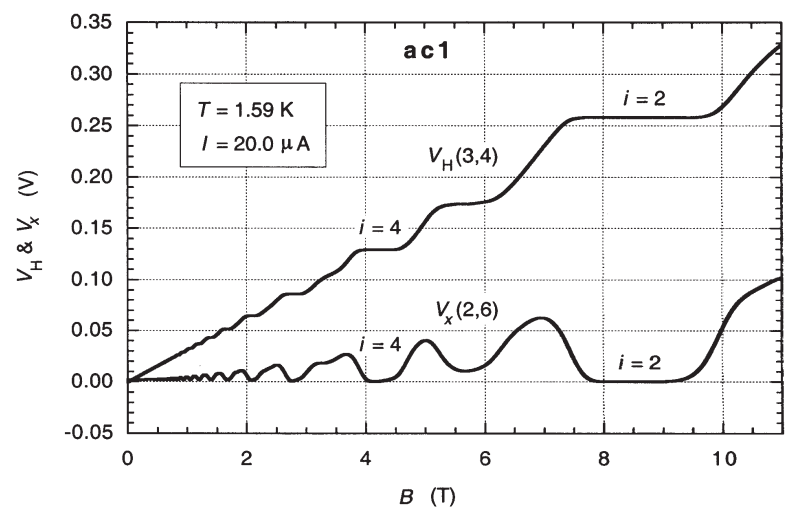

Fig. 1. Magnetic field sweep for the central quantum Hall voltage probes $V_{\mathrm{H}}(3,4)$ and the longitudinal voltage probes $V_{x}(2,6)$ at $I=$ $20.0 \mu \mathrm{A} \mathrm{dc}$ and $T=1.59 \mathrm{~K}$. The magnetic flux density $B$ is in tesla. Voltage probe numbers are identified in Fig. 7.

The device looks promising in this sweep. However, three-terminal contact resistance measurements at the approximate currents that will pass through each contact in the quadruple-series mode on the $i=2(12906.4 \Omega)$ quantum Hall plateau at $8.3 \mathrm{~T}$ and $1.58 \mathrm{~K}$ tell a different story: $\mathrm{S}^{\prime}=9 \Omega, \mathrm{D}^{\prime}=0.04 \Omega, 1^{\prime}=$ $115 \Omega, 2^{\prime}=238 \Omega, 3^{\prime}=1417 \Omega, 4^{\prime}=73 \Omega, 5^{\prime}=179 \Omega$, and $6^{\prime}=7644 \Omega$ after measuring the voltages with both current directions and subtracting lead resistances. Furthermore, the $7644 \Omega$ potential contact $6^{\prime}$ exhibited Corbino-like behavior: its contact resistance increased with decreasing current at small currents due to isolated spikes into the two-dimensional electron fluid, rather than a uniform diffusion. Current circulated around the spikes, as well as along the device. That behavior (pointed-out by K. C. Lee of NIST) would remain undetected if its contact resistance had been measured at the $20.0 \mu \mathrm{A}$ source-drain current, rather than the $\approx 0.01 \mu \mathrm{A}$ quadruple-series current appropriate 
for that probe. It is important to measure contact resistances at the approximate multi-series connection currents, and for each current direction. Resistances of the $1^{\prime}, 3^{\prime}$, and $5^{\prime}$ potential contacts were also currentdependent, but in a more typical way: their resistances increased with increasing current.

The Corbino-like behavior of potential contact $6^{\prime}$ created several problems: static voltages induced when reversing dc current directions or changing ac bridge cables sometimes required minutes or hours to decay (making measurements time-consuming), and the QHR values $R_{\mathrm{H}}=V_{\mathrm{H}} / I$ often fluctuated by about 1 part in $10^{7}$ $\left(1 \times 10^{-7} R_{\mathrm{H}}\right)$ within a day or between days. A device with properties this poor would normally be immediately discarded, but no others were available. The following measurements are intended only as indicators of what could be done with a good device.

The device was cooled from $4.2 \mathrm{~K}$ to either $1.6 \mathrm{~K}$ or $1.5 \mathrm{~K}$ in about $1 \mathrm{~h}$ each measurement day, but it required another $2 \mathrm{~h}$ to stabilize the QHR value. An example is shown in Fig. 2, where $T$ is the temperature of the lower Cernox thermometer, and $X_{\mathrm{M}}$ and $Y_{\mathrm{M}}$ are the in-phase and $90^{\circ}$ out-of-phase ac QHR bridge main signal components (defined in Sec. 6). A 6.5 V change in $X_{\mathrm{M}}$ corresponds to a 1 part in $10^{6}$ change in the QHR value at $1592 \mathrm{~Hz}$; so the thermal stabilization problem is significant. We have no explanation for this puzzling and time-consuming feature. (It did not arise in previous temperature-dependence experiments using a dc sample probe, and was not due to thermal oscillations because the Cernox thermometers above and below the device remained synchronized and their temperatures decreased monotonically. We never filled the variable

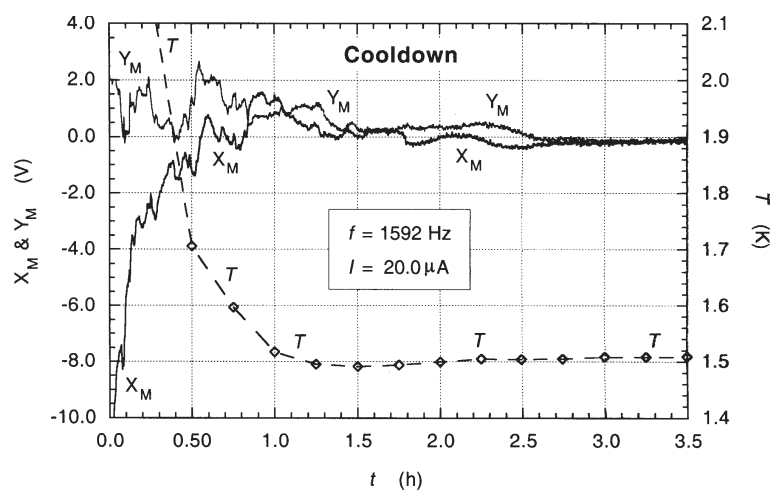

Fig. 2. Typical cooling curves from $4.2 \mathrm{~K}$ each measurement day. $X_{\mathrm{M}}$ and $Y_{\mathrm{M}}$ are the in-phase and $90^{\circ}$ out-of-phase ac QHR bridge main signal components (defined in Sec. 6). $T$ is the temperature of a Cernox thermometer located just below QHR device ac1. A $6.5 \mathrm{~V}$ change in $X_{\mathrm{M}}$ corresponds to a 1 part in $10^{6}$ change in the QHR value at $1592 \mathrm{~Hz}$. temperature insert with smaller heights of liquid helium before pumping to see if that reduced the stabilization time.)

\section{Reference Resistors}

All measurements were made in 1:1 ratio to avoid scaling ambiguities. The main reference standard was a $12906.4 \Omega$ wire-wound resistor labeled $12.9 \mathrm{WW} 1$. It was assembled from Tegam resistor components made of wire wound on mica cards and placed in a shielded container. It was designed for minimum capacitancesto-shield, trimmed to within about 6 parts in $10^{6}$ of the dc QHR value, and maintained in an oil bath at $(25.00 \pm 0.04){ }^{\circ} \mathrm{C}$. This resistor type is used in the ac part of the NIST calculable capacitor chain from the Farad to the Ohm [19]. They are very stable, except for small linear drift rates. The $12.9 \mathrm{WW} 1 \mathrm{drift}$ rate is not yet determined, but typically this type drifts about 1.5 parts in $10^{7}$ per year.

This reference resistor was compared with the $12906.4 \Omega, i=2$ plateau of QHR device ac1, and also with a NL Engineering (Norman Lloyd) quadrifilar resistor (Gibbings [20] resistor) of $12906.4 \Omega$ nominal value that we labeled 12.9QF1. Quadrifilar resistor $12.9 \mathrm{QF} 1$ is maintained in a self-contained air bath and thermally-lagged with additional insulation. It lacks an independent sensor to monitor temperature stability, and looses control when the room temperature exceeds $24.5^{\circ} \mathrm{C}$. Self-heating occurs at currents above $50 \mu \mathrm{A}$. Its resistance value differs from the dc QHR value by about 48 parts in $10^{6}$, and can unpredictably change a few parts in $10^{7}$ over several hours, necessitating computational adjustment to a reference value (which we chose at $1592 \mathrm{~Hz}$ ).

\section{DC Measurements}

It is important to understand the dc properties of the QHR device when making ac measurements; so we begin with those. Contact resistances were discussed in Sec. 3. Figure 3 shows magnetic field sweeps of the $12906.4 \Omega i=2$ plateaus of QHR device ac1 for the three single-series-connected quantum Hall voltage probe sets $V_{\mathrm{H}}(1,2), V_{\mathrm{H}}(3,4)$, and $V_{\mathrm{H}}(5,6)$ at $1.59 \mathrm{~K}$ and $20.0 \mu \mathrm{A}$ dc using digital multimeters to measure the Hall voltages and magnetic flux density $B$. Those sweeps are magnified in Fig. 4 to 4 parts in $10^{6}$ resolution. Measurements involving the $1417 \Omega$ (probe 3 ) and $7644 \Omega$ (probe 6 ) contacts are much noisier (with possible discrete values within the noise). Different offset 


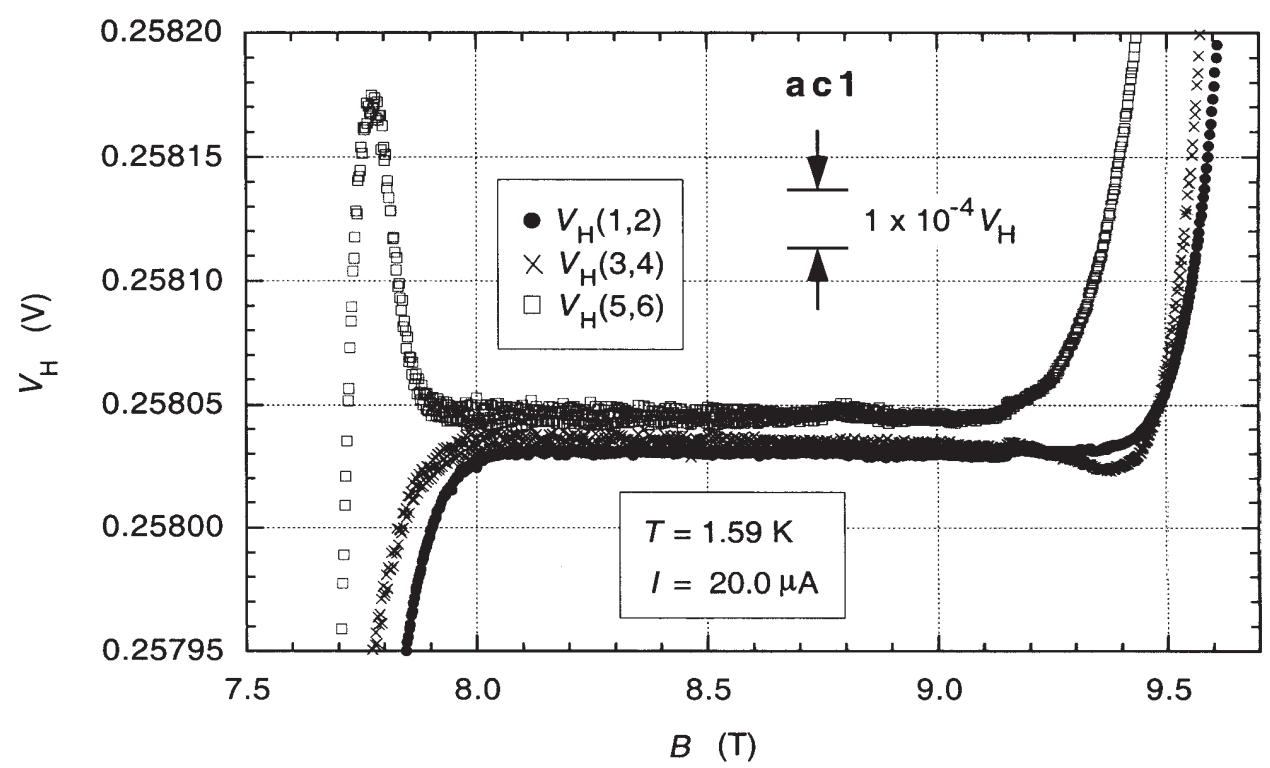

Fig. 3. Magnetic field sweeps of the $12906.4 \Omega i=2$ plateaus of QHR device ac1 for the three single-seriesconnected quantum Hall voltage probe sets $V_{\mathrm{H}}(1,2), V_{\mathrm{H}}(3,4)$, and $V_{\mathrm{H}}(5,6)$ at $1.59 \mathrm{~K}$ and $20.0 \mu \mathrm{Adc}$.

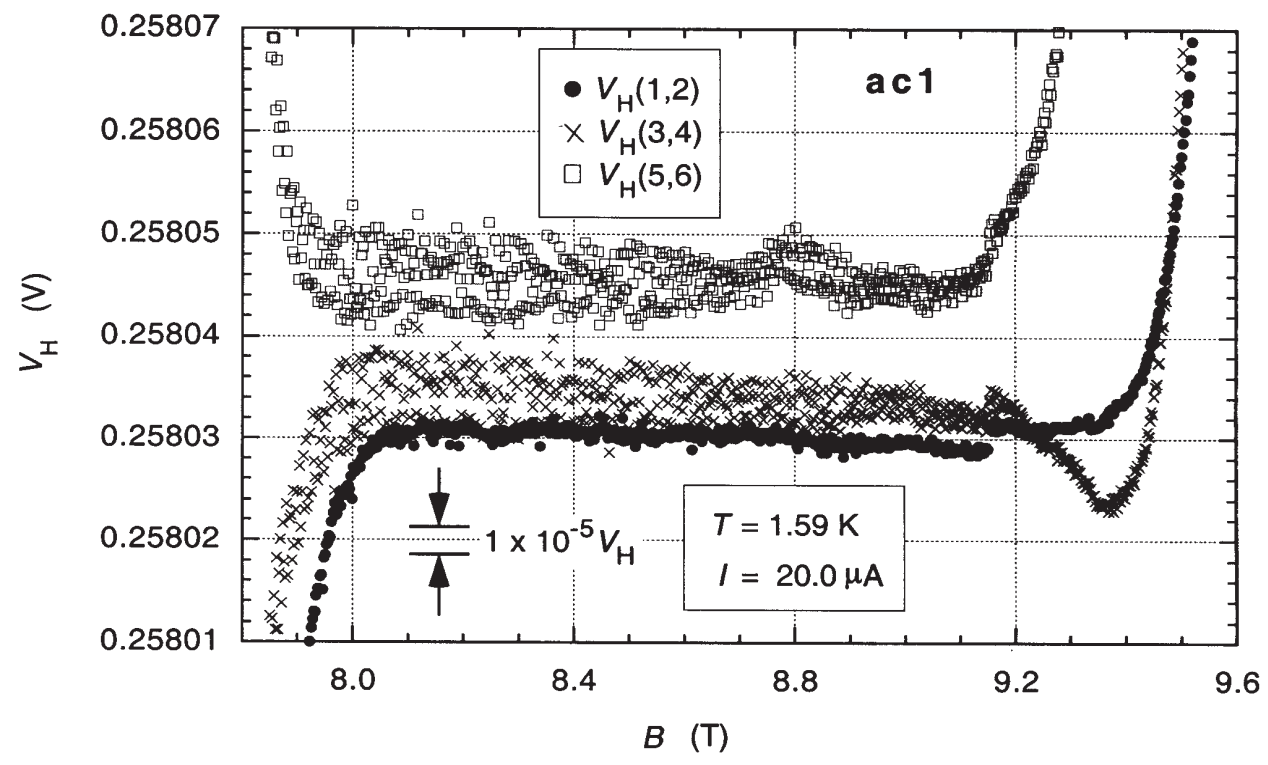

Fig. 4. The magnetic field sweeps of Fig. 3 magnified to 4 parts in $10^{6}$ resolution. 
voltages of the three Hall probe sets probably arise from thermoelectric effects and are of no consequence since we find later in this section that the three $V_{\mathrm{H}}$ values are comparable when including reverse-current measurements.

Large voltage shifts (about 1 part in $10^{5}$ of $V_{\mathrm{H}}$ ) appeared in all three Hall probe sets around $9.15 \mathrm{~T}$ in Fig. 4. They probably resulted from an hourly reading of the liquid helium level of the variable temperature insert into which the sample probe is placed since a reading occurred at that time. Liquid helium level readings were never made during subsequent measurements after daily cooldown from $4.2 \mathrm{~K}$.

Figure 5 shows magnetic field sweeps of the longitudinal voltage probe sets $V_{x}(2,4), V_{x}(4,6)$, and $V_{x}(2,6)$ in the $i=2$ plateau region at $1.59 \mathrm{~K}$ and $20.0 \mu \mathrm{A}$ dc. $V_{x}$ minima involving the $7644 \Omega$ contact resistance of probe 6 were much noisier and narrower, and shifted to smaller magnetic fields. The centroid of the $V_{x}(2,6)$ minima is about $8.3 \mathrm{~T}$.

We measured the $V_{x}$ minima to 1 part in $10^{9}$ type A, $1 \sigma$ uncertainty at $24.0 \mu \mathrm{A} \mathrm{dc}, 1.58 \mathrm{~K}$, and $8.3 \mathrm{~T}$ with an automated potentiometeric system POTSYS [21]. Longitudinal resistance $r_{x}=V_{x} / I$ was negligible for $r_{x}(2,4)$, but $r_{x}(4,6)$ and $r_{x}(2,6)$ were both $0.4 \mathrm{~m} \Omega$. The $7644 \Omega$ probe 6 contact resistance clearly has an effect on $r_{x}$. ( $V_{x}$ measurements would have also been made on the high-voltage side of the device if it had been standards quality.)
The three single-series-connected $R_{\mathrm{H}}=V_{\mathrm{H}} / I \quad i=2$ quantized Hall resistances $R_{\mathrm{H}}(1,2), R_{\mathrm{H}}(3,4)$, and $R_{\mathrm{H}}(5,6)$ of QHR device ac1 were compared with the $12906.4 \Omega$ wire-wound resistor $12.9 \mathrm{WW} 1$ at $I=29.1 \mu \mathrm{A} \mathrm{dc}$, $T=1.58 \mathrm{~K}$, and $B=8.3 \mathrm{~T}$ using an automated measurement system that reverses currents, exchanges positions of two digital multimeters, and exchanges the positions of ac1 and $12.9 \mathrm{WW} 1$ in the bridge. The type A, $1 \sigma$, ac1/12.9WW1 ratio values for the three single-series measurements differed from unity by $(-5.97 \pm 0.02)$ $\times 10^{-6},(-5.97 \pm 0.02) \times 10^{-6}$, and $(-5.91 \pm 0.02) \times 10^{-6}$. The device therefore seems homogeneous, with the possible exception of $R_{\mathrm{H}}(5,6)$ which involves the $7644 \Omega$ contact. We note, however, that none of these three measurement sets were repeated, and we sometimes observed shifts and decays after switching. Also, we later found in the ac measurements that the $R_{\mathrm{H}}$ values were stable and reproducible only to within 1 part in $10^{7}$ because of the Corbino effect. Therefore we assign uncertainties of $\pm 0.1 \times 10^{-6}$ to these measurements, and assume homogeneity only within that uncertainty. (DC cryogenic current comparator measurements on a good device would allow several parts in $10^{9}$ uncertainties.)

Cage, Jeffery, and Matthews [16] predicted that devices with two sets of external quadruple-series connections allow dc guideline properties and $\mathrm{dc}$ and ac QHR values with small and predicable quadratic frequency dependences to be measured with all sample

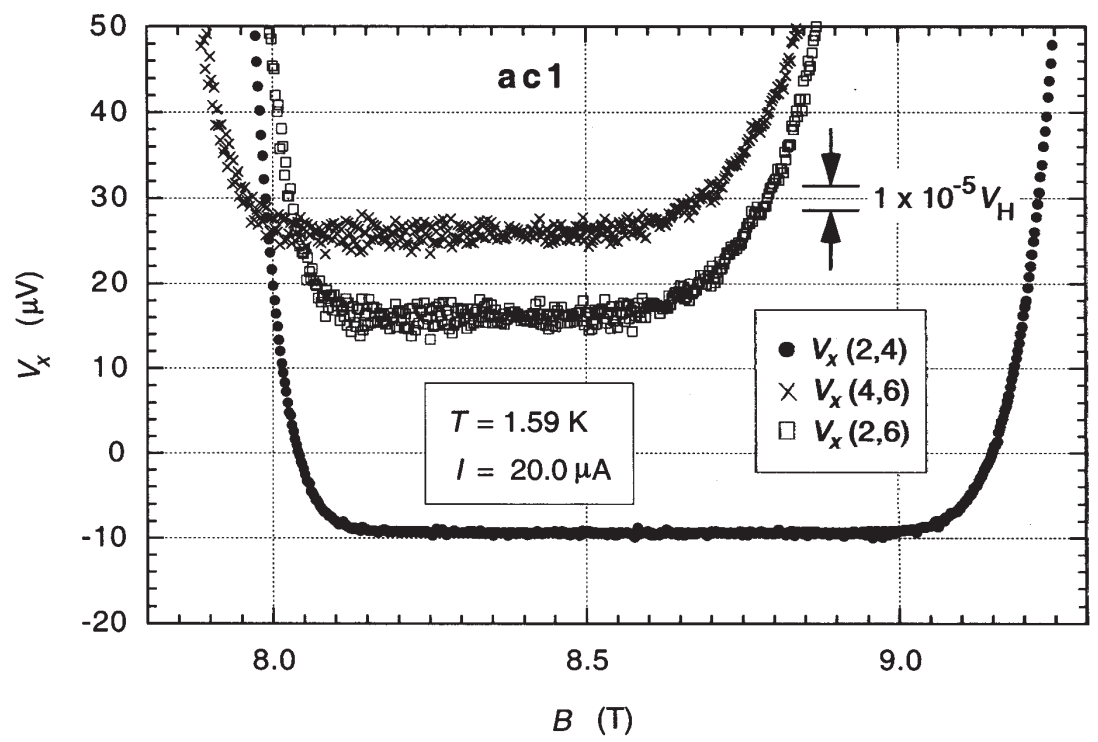

Fig. 5. Magnetic field sweeps of the longitudinal voltage probe sets $V_{x}(2,4), V_{x}(4,6)$, and $V_{x}(2,6)$ in the $i=2$ plateau region at $1.59 \mathrm{~K}$ and $20.0 \mu \mathrm{A} \mathrm{dc}$. 
probe leads attached at the device. Figure 6 is a magnetic field sweep of the quadruple-series-connected $i=2$ plateau $V_{\mathrm{H}}(\mathrm{Y}, \mathrm{Z})$ at $20.0 \mu \mathrm{A} \mathrm{dc}$ and $1.58 \mathrm{~K}$, where room temperature locations $\mathrm{Y}$ and $\mathrm{Z}$ are defined in Fig. 7. The plateau appears flat in this 1 part in $10^{6}$ resolution plot. However, the 1 part in $10^{8}$ resolution plot of Fig. 12 that will be shown for ac currents suggests the dc "plateau" is likely an inverted "U", with no flat region. Furthermore, Fig. 12 looks like Fig. 6 when plotted at 1 part in $10^{6}$ resolution. DC cryogenic current comparator (CCC) measurements would have the resolution to confirm this inverted " $U$ " supposition, but it was not worth moving CCC apparatus between laboratories for this poor-quality device. (CCC measurements would have been made across the plateau regions of $V_{\mathrm{H}}(\mathrm{Y}, \mathrm{Z}), V_{\mathrm{H}}(1,2), V_{\mathrm{H}}(3,4)$, and $V \mathrm{H}(5,6)$ to several parts in $10^{9}$ resolution on a good device.)

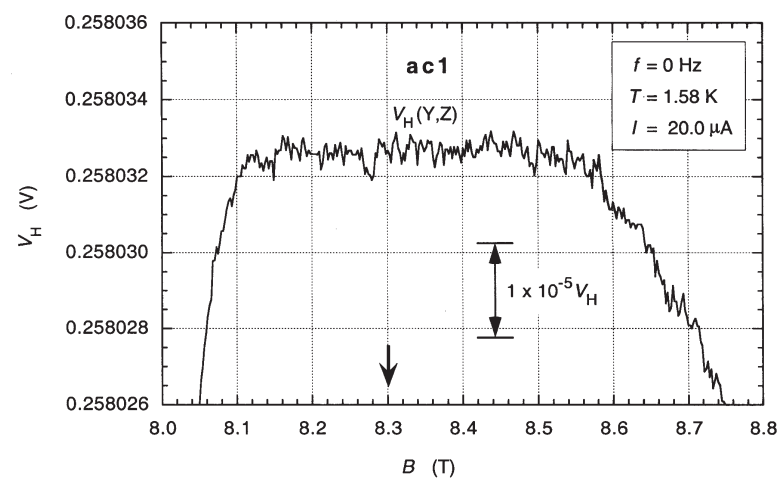

Fig. 6. Magnetic field sweep of the quadruple-series-connected $i=2$ plateau $V_{\mathrm{H}}(\mathrm{Y}, \mathrm{Z})$ at $20.0 \mu \mathrm{A}$ dc and $1.58 \mathrm{~K}$. Star locations $\mathrm{Y}$ and $\mathrm{Z}$ are defined in Fig. 7. The arrow at $B=8.3 \mathrm{~T}$ is the centroid of the $V_{x}(2,6)$ minimum.

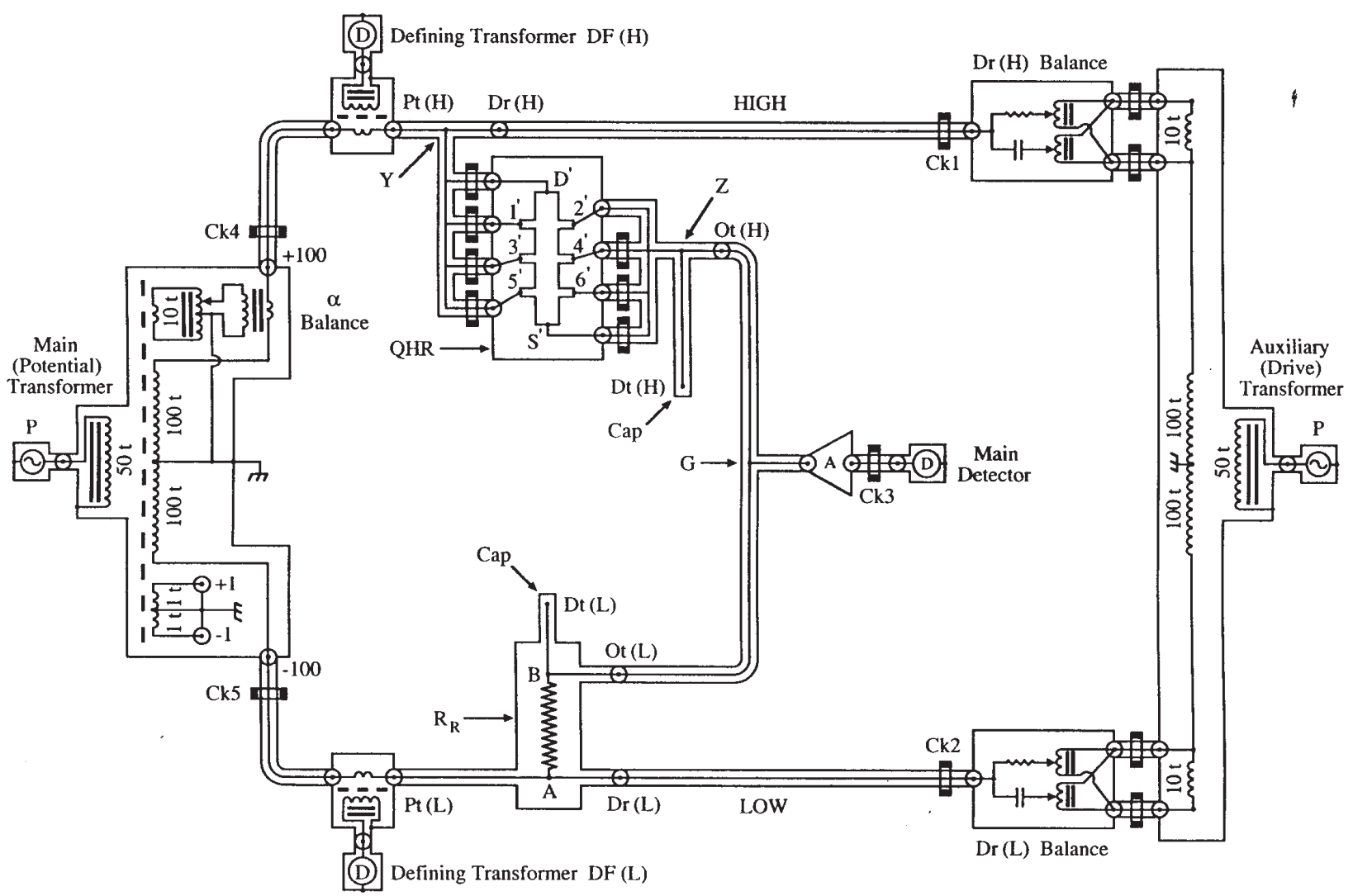

Fig. 7. Simplified representation of the NIST multifrequency transformer ratio bridge for 1:1 ratio measurements in the three-terminal-pair (3TP) mode. 
We see from Eq. (55) of Ref. [16] that $V_{\mathrm{H}}(\mathrm{Y}, \mathrm{Z})$ is primarily the quantity $V_{\mathrm{H}}(3,4)-V_{x}(2,6)$ in homogeneous, quadruple-series-connected devices. That is of no consequence in good devices when cooled to temperatures where $V_{x}(2,6)$ is negligible over the $V_{x}$ minima region. But $V_{x}(2,6)$ is not negligible here because of poor contacts. That may be the source of the possible inverted "U" shape of $V_{\mathrm{H}}(\mathrm{Y}, \mathrm{Z})$.

The quadruple-series-connected resistance $R_{\mathrm{H}}(\mathrm{Y}, \mathrm{Z})$ was compared with the $12906.4 \Omega$ wire-wound reference resistor $12.9 \mathrm{WW} 1$ at $I=29.1 \mu \mathrm{A} \mathrm{dc}, T=1.58 \mathrm{~K}$, and $B=8.3 \mathrm{~T}$ using the automated double-multimeter measurement system. The ratio differed from unity by $(-6.03 \pm 0.02) \times 10^{-6}$. That value is slightly smaller than the three single-series ratios because the quadruple-series mode measures $R_{\mathrm{H}}-\left[r_{x}(2,4)+r_{x}(4,6)\right]$ [16] and the value of $r_{x}(4,6)$ is $3 \times 10^{-8} R_{\mathrm{H}}$. The dc measurements would have been repeated at least once, and at different places along the plateau, but programmatic constraints interceded. As mentioned earlier, we found during subsequent ac measurements for this device that the QHR values were stable and reproducible only to within \pm 1 part in $10^{7}$ because of the Corbino effect. Therefore we assign a dc value of $\left[1-(6.0 \pm 0.1) \times 10^{-6}\right]$ to this 1:1 ratio. No effort was made to see how well the $i=2 \mathrm{dc}$ quadruple-series QHR value of this poorquality device approximates the $12906.4035 \Omega$ von Klitzing constant $R_{\mathrm{K}}$ since we were only investigating convergence of ac and dc values in this experiment, and would in any case not use ac1 as a standard.

\section{AC Bridge}

The NIST multifrequency transformer bridge can measure 1:1, 2:1, and 10:1 ratios. We used interchanged 1:1 ratios here to minimize ambiguities, and the same set of bridge windings in every interchanged measurement. Figure 7 shows a simplified representation of the three-terminal-pair (3TP) mode in 1:1 ratio. ("Terminal-pair" is an accessible coaxial connection (port) consisting of an inner conductor and its shield. "Three" is the number of terminal-pair connections of a 4-port standard that meet the 4TP balance conditions [18].)

A $20.0 \mu \mathrm{A}$ rms drive current, generated by primary voltage source signal $\mathrm{P}$ and an auxiliary (drive) transformer, passes through: coaxial cable on the High $(\mathrm{H})$ side of the bridge to drive port $\operatorname{Dr}(\mathrm{H})$ at external "star" connector Y; QHR device ac1 (connected in quadrupleseries between external stars $\mathrm{Y}$ and $\mathrm{Z}$; coaxial cables between out ports $\mathrm{Ot}(\mathrm{H})$ and $\mathrm{Ot}(\mathrm{L})$ on the High and Low (L) sides of the bridge; reference resistor $12.9 \mathrm{WW} 1$ (shown here as resistor $R_{\mathrm{R}}$, with internal connection points $\mathrm{A}$ and $\mathrm{B})$ to drive port $\operatorname{Dr}(\mathrm{L})$; and back to the auxiliary transformer. Passive coaxial chokes [22] (current equalizers) Ck1 and Ck2 (with 20 turns wound around a magnetic core) assure nearly equal and opposite currents in the inner and outer conductors of the coaxial drive cables.

The primary voltage signal $\mathrm{P}$ is also supplied to the main (potential) transformer. Main balance is achieved by adjusting the six-decade in-phase $\alpha$ dials and $90^{\circ}$ out-of-phase $\beta$ dials on the main (potential) transformer until the in-phase and out-of-phase impedance signal components $X_{\mathrm{M}}$ and $Y_{\mathrm{M}}$ are amplified at A and nulled in the main lock-in detector $\mathrm{D}$. There is negligible current in the inner conductor to the main detector at balance. Coaxial choke $\mathrm{Ck} 3$ then assures negligible current in the outer conductor. The inner conductor of "star" connector $\mathrm{G}$ is at virtual ground at balance. (The $\beta$ adjustment network is not shown in the figure. It consists of a six-decade $\beta$ balance identical to the $\alpha$ balance, a $10 \mathrm{nF}$ mica capacitor in a $25^{\circ} \mathrm{C}$ oil bath, and a choked coaxial cable also inserted into star G.)

Defining transformers $\mathrm{DF}(\mathrm{H})$ and $\mathrm{DF}(\mathrm{L})$, and lock-in detectors $\mathrm{D}$ assure negligible current in the inner conductors at the High and Low potential ports $\mathrm{Pt}(\mathrm{H})$ and $\mathrm{Pt}(\mathrm{L})$ by adjusting four-decade in-phase and out-ofphase inductive voltage dividers (IVDs) $\operatorname{Dr}(\mathrm{H})$ Balance and $\operatorname{Dr}(\mathrm{L})$ Balance in the drive circuit to null the inphase and out-of-phase defining transformer detector signals $\mathrm{DF}(\mathrm{H})$ and $\mathrm{DF}(\mathrm{L})$. Coaxial chokes $\mathrm{Ck} 4$ and $\mathrm{Ck} 5$ in the potential circuit approximate the zero current condition in the outer conductors at potential ports $\mathrm{Pt}(\mathrm{H})$ and $\mathrm{Pt}(\mathrm{L})$. Brass caps cover the outer conductors of the open detection ports $\operatorname{Dt}(\mathrm{H})$ and $\operatorname{Dt}(\mathrm{L})$ to minimize noise.

Systematic errors may arise if the network is overchoked or under-choked. No effort has yet been made to vary the number or location of chokes. We chose the coaxial cable between contact $2^{\prime}$ and star connector $\mathrm{Z}$ to be unchoked to avoid over-choking the QHR "standard" itself because that cable is near ground potential and carries the smallest current. The outer shields of all bridge components are grounded via one point at the main (potential) transformer. (The effect of adding extra ground connections will be tested in the future.)

This circuit satisfies the 3TP conditions at both the QHR "standard" and the reference resistor: (1) negligible current at the inner and outer conductors of potential ports $\mathrm{Pt}(\mathrm{H})$ and $\mathrm{Pt}(\mathrm{L})$, and therefore no inphase currents in the potential cables to external connection star Y of the QHR "standard" or to internal con- 
nection point A of the reference resistor; (2) negligible current and voltage in the inner and outer conductors to the main detector D; (3) the same current to/from the QHR "standard" and the reference resistor at connection star $\mathrm{G}$ (where the inner conductor is at virtual ground at balance); and (4) equal and opposite currents in the inner and outer conductors of cables between external connection star Z of the QHR "standard" and internal connection point $\mathrm{B}$ of the resistor. (In addition, detection ports $\mathrm{Dt}(\mathrm{H})$ and $\mathrm{Dt}(\mathrm{L})$ have no current since they are open-circuited. That meets part of the 4TP definition. However, ports $\mathrm{Dt}(\mathrm{H})$ and $\mathrm{Dt}(\mathrm{L})$ are not at ground potential because of cable loses to star G.)

The 3TP circuit of Fig. 7 works perfectly well, but Fig. 8 shows modifications to minimize cabling changes between the 3TP and 4TP measurement modes. Voltage injectors $\mathrm{VI}(\mathrm{H})$ and $\mathrm{VI}(\mathrm{L})$ were added to the coaxial cables leaving the out ports $\operatorname{Ot}(\mathrm{H})$ and $\mathrm{Ot}(\mathrm{L})$, and the VI output windings were shorted. This only increased cable impedances from each out port
$\operatorname{Ot}(\mathrm{H})$ or $\mathrm{Ot}(\mathrm{L})$ to star $\mathrm{G}$ by $0.5 \mathrm{~m} \Omega, 4.4 \mathrm{pF}$, and $1 \mu \mathrm{H}$ (The purposes of IVDs $\mathrm{Ot}(\mathrm{H})$ Balance and $\mathrm{Ot}(\mathrm{L})$ Balance and their capped cables will be explained for the 4TP mode. They have no influence on 3TP measurements.)

3TP balances are made in an iterative process using amplifier A, lock-in detector $\mathrm{D}$, and the bridge main adjustment dials $\alpha$ and $\beta$ to null the $X_{\mathrm{M}}$ and $Y_{\mathrm{M}}$ impedance signals at star G. A second lock-in detector D moves between defining transformers $\mathrm{DF}(\mathrm{H})$ and $\mathrm{DF}(\mathrm{L})$ to assure negligible currents in the inner conductors at potential ports $\mathrm{Pt}(\mathrm{H})$ and $\mathrm{Pt}(\mathrm{L})$ by adjusting IVDs $\operatorname{Dr}(\mathrm{H})$ Balance and $\operatorname{Dr}(\mathrm{L})$ Balance. This second detector is always replaced with a shorted inner/outer connector at the $\mathrm{DF}(\mathrm{H})$ or $\mathrm{DF}(\mathrm{L})$ output winding, and is completely removed on final bridge balance.

We initially inserted a "combining network" like that of Fig. 6 in Cutkosky's paper [18] between ports Ot(H), $\operatorname{Dt}(\mathrm{H})$ and ports $\mathrm{Ot}(\mathrm{L}), \operatorname{Dt}(\mathrm{L})$ to approximate the 4TP definition. (An example is shown in Fig. 3 of Ref. [23]

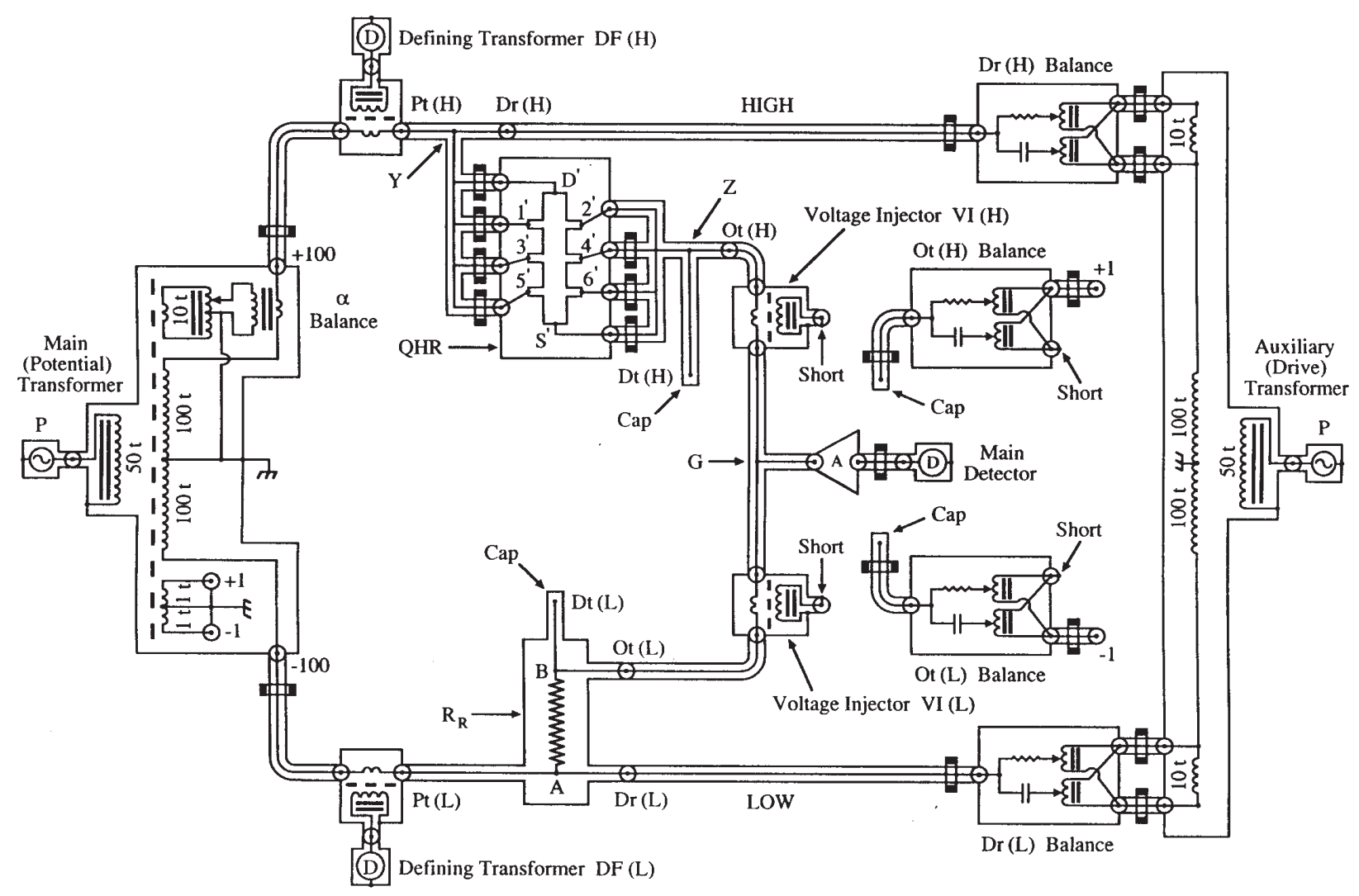

Fig. 8. Modifications of the 3TP circuit in Fig. 7 to minimize cabling changes between 3TP and 4TP measurement modes. 
for an earlier NIST bridge.) However, measurement errors arose that were sometimes as large as 18 parts in $10^{6}$ under "proper" 4TP balance conditions using different internal resistance values of the combining network when measuring 10:1 resistance ratios. (Perhaps this resulted from using out-of-phase $\beta$ signal injection into a ground potential location of the combining network, rather than into a location on the higher potential side. This will be checked in future.)

We therefore removed the combining network and made all 4TP measurements by "brute force" using five in-phase and five out-of-phase balances, as indicated in Fig. 9. Injectors $\mathrm{VI}(\mathrm{H})$ and $\mathrm{VI}(\mathrm{L})$ insert voltages into the coaxial cables between out ports $\operatorname{Ot}(\mathrm{H})$ and $\mathrm{Ot}(\mathrm{L})$ to make up for cable loses such that the in-phase and out-of-phase signals are nulled at detection ports $\mathrm{Dt}(\mathrm{H})$ and $\operatorname{Dt}(\mathrm{L})$, as well as at star G. This is accomplished with IVD adjustments $\mathrm{Ot}(\mathrm{H})$ Balance and $\mathrm{Ot}(\mathrm{L})$ Balance to null the in-phase and out-of-phase signals at ports $\operatorname{Dt}(\mathrm{H})$ and $\mathrm{Dt}(\mathrm{L})$ using amplifiers $\mathrm{A}$ and lock-in detectors $\mathrm{D}$. Thus the inner conductors of ports $\mathrm{Dt}(\mathrm{H})$,
$\operatorname{Dt}(\mathrm{L})$, and $\mathrm{G}$ are all at virtual ground at balance. Those two IVDs are connected to +1 turn and -1 turn taps on a separate winding of the main (potential) transformer. (It is possible that they slightly load the potential transformer. That will be tested in future by connecting them to existing +1 and -1 or +10 and -10 taps (not shown) of the drive transformer.)

Cable lengths were carefully matched to minimize errors. Inner and outer contacts of every British Post Office (BPO) cable connector were individually tested and polished to avoid ratio measurement shifts of order 1 part in $10^{7}$. (All outer contacts of a recent BPO purchase were too loose, and the inner contacts very tight; so the connections "felt" good, but occasionally caused large shifts.) Each week we twisted every connector several times to maintain polish and avoid shifts of order 1 part in $10^{8}$. Leakage resistances of all BPO connectors, the few BNC connectors, and all coaxial cables are greater than $10^{14} \Omega$. They were maintained to that value with dust covers on all open connectors.

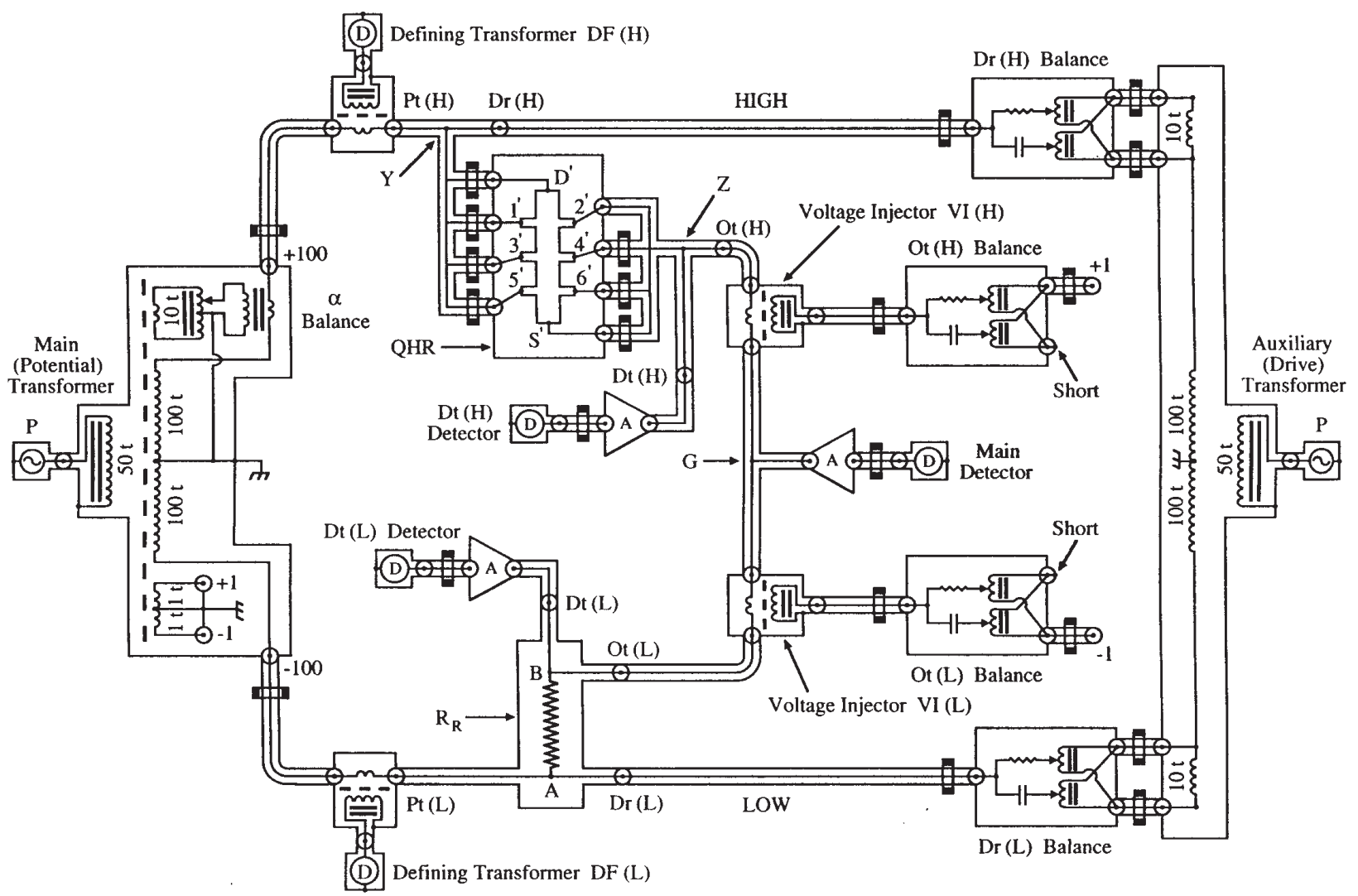

Fig. 9. NIST multifrequency bridge circuit representation for 1:1 ratio measurements in the 4TP mode. 
4TP balances use the 3TP procedures listed above to obtain a preliminary main balance and null currents at potential ports $\operatorname{Pt}(\mathrm{H})$ and $\operatorname{Pt}(\mathrm{L})$. Amplifier $\mathrm{A}$ and lock-in detector $\mathrm{D}$, used for nulling the $X_{\mathrm{M}}$ and $Y_{\mathrm{M}}$ signals at main balance star $\mathrm{G}$, is then sequentially moved to detector ports $\mathrm{Dt}(\mathrm{H})$ and $\mathrm{Dt}(\mathrm{L})$, and their inphase and out-of-phase signals nulled by adjusting IVDs $\mathrm{Ot}(\mathrm{H})$ Balance and $\mathrm{Ot}(\mathrm{L})$ Balance. (Brass caps cover any open outer conductors at ports $\operatorname{Dt}(\mathrm{H}), \mathrm{Dt}(\mathrm{L})$, and star $\mathrm{G}$ to minimize noise.)

It requires several iterations and some experience to simultaneously null all three sets of low voltage inphase and out-of-phase signals because the main, $\mathrm{Ot}(\mathrm{H})$, and $\mathrm{Ot}(\mathrm{L})$ balance adjustments interact. Although tedious to initially determine at each frequency, the bridge adjustment parameters are reproducible, and should provide a close approximation to ideal 4TP measurements.

The 4TP bridge, at balance, measures the ratio of the QHR "standard" (with its in-phase impedance component defined between external connection stars $\mathrm{Y}$ and $\mathrm{Z}$ ) and the wire-wound resistor (defined between internal connection points A and B). (Figure 4 of the Cage, Jeffery, and Matthews equivalent circuit model [16] defines the QHR "standard" at access ports $\operatorname{Dr}(\mathrm{H})$, $\mathrm{Pt}(\mathrm{H}), \operatorname{Dt}(\mathrm{H})$, and $\mathrm{Ot}(\mathrm{H}) \cdot \operatorname{Dr}(\mathrm{H}), \operatorname{Dt}(\mathrm{H})$, and $\mathrm{Ot}(\mathrm{H})$ are located on stars $\mathrm{Y}$ and $\mathrm{Z}$. $\mathrm{Pt}(\mathrm{H})$ is separated from star $\mathrm{Y}$ by a small length of coaxial cable. The additional impedances from stars $\mathrm{Y}$ and $\mathrm{Z}$ to those four access ports have negligible effect on the in-phase component of the ac QHR value in our bridge at 4TP balance.)

Figure 10 demonstrates the bridge sensitivity of the balanced and amplified in-phase main signal component $X_{\mathrm{M}}$ for the ac1/12.9WW1 ratio with a -1 part in $10^{7}$ change of the dial settings. The bridge has a 5 parts in $10^{9}$ resolution at $1592 \mathrm{~Hz}$ and $20.0 \mu \mathrm{A}$. This is

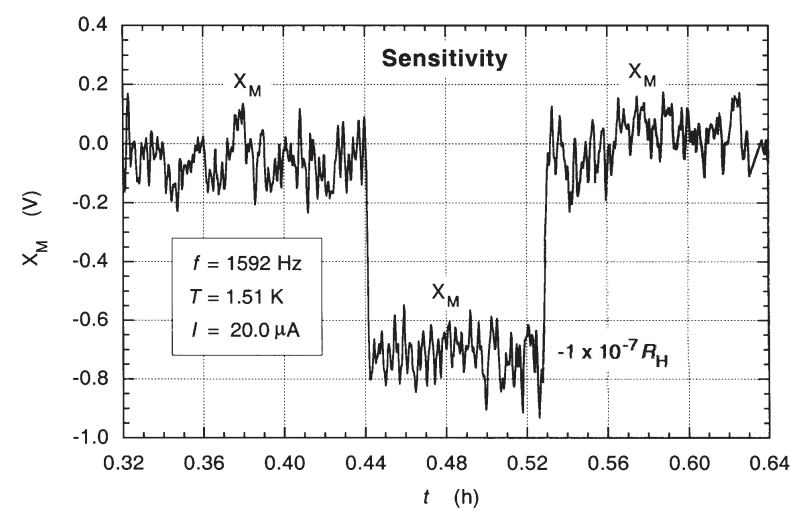

Fig. 10. NIST multifrequency bridge sensitivity of the main inphase impedance signal component $X_{\mathrm{M}}$ for the ac1/12.9WW1 ratio with a -1 part in $10^{7}$ change of the $\alpha$ dials setting. comparable with any bridge in the NIST calculable capacitor chain from the Farad to the Ohm [19]. Its inphase resolution increases at lower frequencies and decreases at higher frequencies.

\section{AC QHR Measurements}

Figure 11 shows a magnetic field sweep over the quadruple-series-connected $i=2$ plateau $V_{\mathrm{H}}(\mathrm{Y}, \mathrm{Z})$ at $1.51 \mathrm{~K}$ compared in 4TP mode with the $12.9 \mathrm{k} \Omega$ wirewound resistor $12.9 \mathrm{WW} 1$ at $20.0 \mu \mathrm{A}$ rms and $1592 \mathrm{~Hz}$. The $90^{\circ}$ out-of-phase signal component $Y_{\mathrm{M}}$ is offset for clarity from the in-phase main lock-in detector signal output $X_{\mathrm{M}} \cdot X_{\mathrm{M}}$ and $Y_{\mathrm{M}}$ sweeps at $700 \mathrm{~Hz}, 3000 \mathrm{~Hz}$, and $5000 \mathrm{~Hz}$ have similar shapes and magnetic field locations. Figure 12 shows the Fig. $11 X_{\mathrm{M}}$ signal magnified. The ac QHR "plateau" is an inverted "U" with no

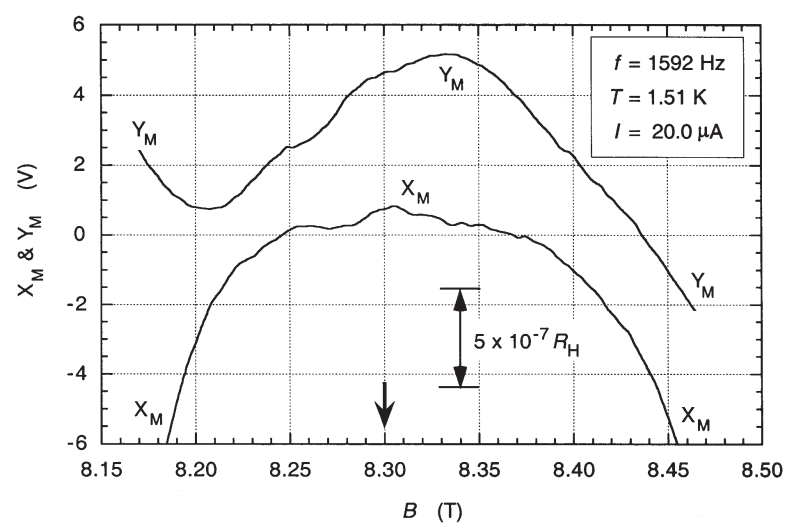

Fig. 11. Magnetic field sweep over the quadruple-series-connected $i=2$ plateau $V_{\mathrm{H}}(\mathrm{Y}, \mathrm{Z})$ at $1.51 \mathrm{~K}$ compared in $4 \mathrm{TP}$ mode with wirewound resistor $12.9 \mathrm{WW} 1$ at $20.0 \mu \mathrm{A} \mathrm{rms}$ and $1592 \mathrm{~Hz}$. The $90^{\circ}$ outof-phase signal component $Y_{\mathrm{M}}$ is offset for clarity from the in-phase main lock-in detector signal component $X_{\mathrm{M}}$. The arrow at $B=8.3 \mathrm{~T}$ is the centroid of the $V_{x}(2,6)$ minimum.

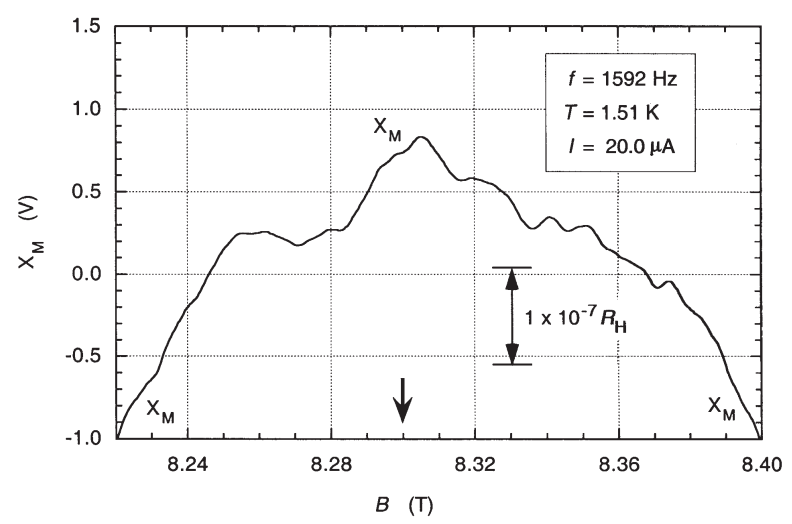

Fig. 12. Magnified version of the Fig. $11 X_{\mathrm{M}}$ signal. 
flat region. (As mentioned before in Sec. 5, it appears just as flat as the dc quadruple-series plateau of Fig. 6 when plotted to that resolution.) The "peak" feature near $8.3 \mathrm{~T}$ was reproducible, and occurs at the same magnetic flux density as the $V_{x}(2,6)$ minimum. We suspect it is due to the $\left[V_{\mathrm{H}}-V_{x}(2,6)\right]$ aspect of quadrupleseries measurements, and would not appear on plateaus of good devices when cooled enough that $V_{x}(2,6)$ was negligible. This feature may contribute to the \pm 1 part in $10^{7}$ fluctuations in the resistance ratio. AC bridge measurements were always made at the $B=8.3 \mathrm{~T}$ value used for dc measurements.

3TP and 4TP plots of $\alpha$ vs $f$ in Fig. 13 at $20.0 \mu \mathrm{A}$ $\mathrm{rms}, 8.3 \mathrm{~T}$, and $1.51 \mathrm{~K}$ are the in-phase six-decade main dial readings of the resistance ratio ac1/12.9WW1 vs frequency when the device and reference resistor are in their "normal" positions, with ac1 on the High side of the bridge and $12.9 \mathrm{WW} 1$ on the Low side. The bridge is constructed such that: a perfect unity ratio, with no frequency dependences or bridge corrections, would be $\alpha=555.555$, where a change of +1.0 in the $\alpha$ dials reading represents $\mathrm{a}+1.0$ part in $10^{6}$ shift in the inphase ratio signal $X_{\mathrm{M}}$. (We deliberately plot this "raw data" in that format to indicate that no bridge or cable corrections are applied at this stage.) 3TP balances were achieved between $350 \mathrm{~Hz}$ and $5500 \mathrm{~Hz} ; 4 \mathrm{TP}$ balances between $700 \mathrm{~Hz}$ and $5000 \mathrm{~Hz}$. (A $5500 \mathrm{~Hz}$ 4TP datum point was not taken when it appeared for a while that the project was going to be terminated.)

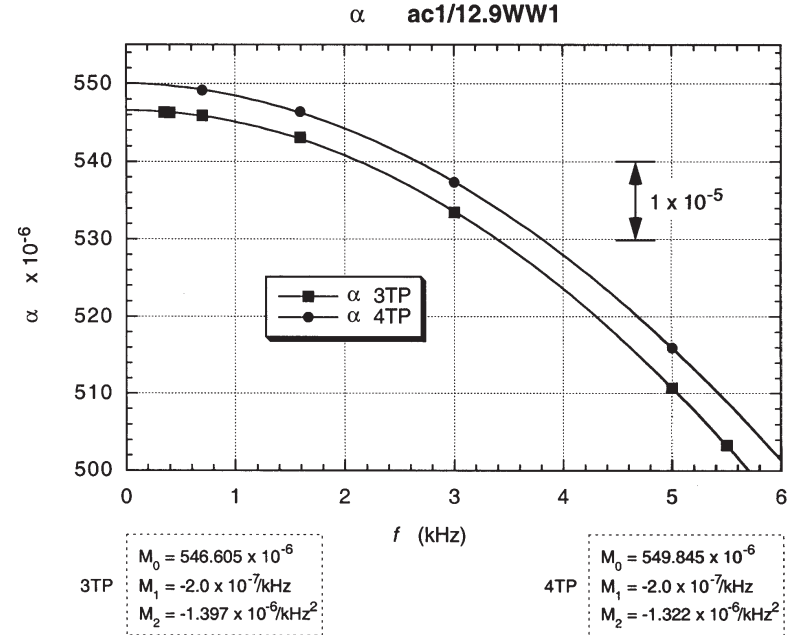

Fig. 13. $3 \mathrm{TP}$ and $4 \mathrm{TP}$ plots of main bridge dial "raw data" readings $\alpha$ vs frequency $f$ at $20.0 \mu \mathrm{A} \mathrm{rms}, 8.3 \mathrm{~T}$, and $1.51 \mathrm{~K}$ when the device and reference resistor are in their "normal" positions, with ac1 on the High side of the bridge and 12.9WW1 on the Low side. A perfect unity ratio, with no frequency dependences or bridge corrections, would be $\alpha=555.555$. A change of +1.0 in the $\alpha$ dials reading represents $\mathrm{a}+1.0$ part in $10^{6}$ shift in the in-phase resistance ratio signal $X_{\mathrm{M}}$
Corbino-like behavior of the $7644 \Omega$ potential contact meant that static voltages induced when changing bridge leads sometimes required minutes, or even hours, to decay. As a result, every measurement has a \pm 1 part in $10^{7}$ uncertainty; which is frustrating when compared with the 5 parts in $10^{9}$ resolution.

Second-order polynomial fits to the data in Fig. 13 assume a constant term $M_{0}$, a linear frequency term $M_{1} f$, and a quadratic frequency term $M_{2} f^{2}$. Coefficients $M_{0}$, $M_{1}$, and $M_{2}$ are listed in the figure. Large linear and quadratic frequency dependences occur for both $3 \mathrm{TP}$ and 4TP measurements.

Figure 14 shows the 3TP and 4TP main dial "raw data" readings $\alpha_{\mathrm{e}}$ vs $f$ with the device and reference resistor positions "exchanged" in the 1:1 ratio measurements: $12.9 \mathrm{WW} 1$ on the High side of the bridge and ac1 on the Low side. We tried two exchange methods: (1) physically moving cables between the $\operatorname{Dr}(\mathrm{H}), \operatorname{Pt}(\mathrm{H})$, $\operatorname{Ot}(\mathrm{H})$ ports and the $\operatorname{Dr}(\mathrm{L}), \operatorname{Pt}(\mathrm{L}), \operatorname{Ot}(\mathrm{L})$ ports; and (2) exchanging the other end of those coaxial cables at the High and Low taps of the main (potential) and auxiliary (drive) transformers. Both methods gave similar results. The later exchange method was used because it is physically easier and involves less change in relative cable positions. There is a large $(6.0 \pm 0.1) \times 10^{-7} / \mathrm{kHz}$ difference in linear frequency dependence between the "exchanged" and "normal" position fits of Figs. 13 and 14 .

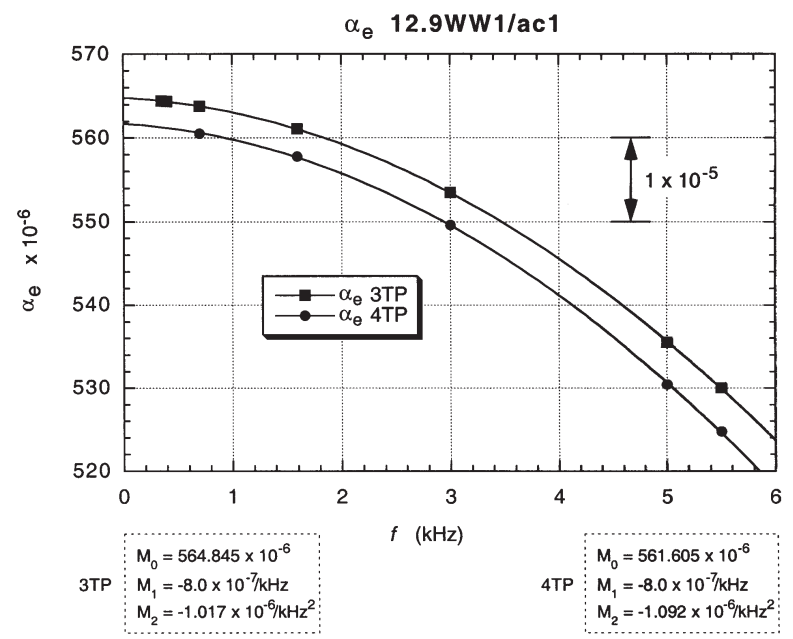

Fig. 14. Main dial 3TP and 4TP "raw data" readings $\alpha_{\mathrm{e}}$ vs $f$ with the device and reference resistor positions "exchanged" in 1:1 ratio measurements: $12.9 \mathrm{WW} 1$ on the High side of the bridge and ac1 on the Low side. 
The in-phase ac bridge contributions $\alpha_{0}$ to the $\alpha$ and $\alpha_{\mathrm{e}}$ measurements of Figs. 13 and 14 are obtained by averaging the sum of the "normal" and "exchanged" "raw data" at each frequency to obtain interchanged bridge in-phase components $\alpha_{0}$ :

$$
\alpha_{0}=\left(\alpha+\alpha_{\mathrm{e}}\right) / 2 \text {. }
$$

Figure 15 plots these $\alpha_{0}$ vs $f$ results, which are the same for both 3TP and 4TP measurements. There is a significant offset from the ideal unity value: $(555.725-555.555) \times 10^{-6}=(+1.7 \pm 1.0) \times 10^{-7}$. Note also the large $(-5.0 \pm 0.1) \times 10^{-7} / \mathrm{kHz}$ linear frequency dependence. A linear dependence term was unexpected, but definitely exists given the \pm 1 part in $10^{7}$ data uncertainties. The $(-1.207 \pm 0.010) \times 10^{-6} / \mathrm{kHz}^{2}$ quadratic dependence is very large.

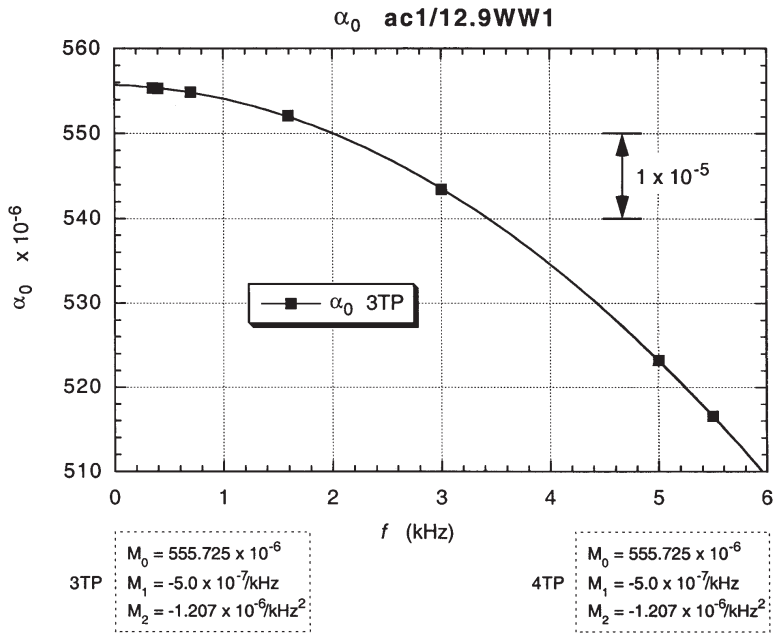

Fig. 15. In-phase ac bridge contributions $\alpha_{0}$ to the $\alpha$ and $\alpha_{\mathrm{e}}$ measurements of Figs. 13 and 14, obtained by averaging the sums of the "normal" and "exchanged" "raw data". These $\alpha_{0}$ vs $f$ results are the same for both 3TP and 4TP measurements.

Interchanged in-phase deviations $\Delta$ from the unity resistance ratio are obtained by averaging the difference between the "normal" and "exchanged" "raw data" for each frequency:

$$
\Delta=\left(\alpha-\alpha_{\mathrm{e}}\right) / 2,
$$

as shown in Fig. 16. Note that the 4TP interchanged ac QHR deviation measurements converge within the \pm 1 part in $10^{7}$ relative standard deviation uncertainty to the dc value of $(-6.0 \pm 0.1)$ parts in $10^{6}$, whereas the interchanged 3TP measurements are in error by $(-3.2 \pm 0.1)$ parts in $10^{6}$ on extrapolation to dc when using the "raw data" with no cable corrections. Cable corrections are not necessary in our 4TP bridge of
Fig. 9 because voltage injectors $\mathrm{VI}(\mathrm{H})$ and $\mathrm{VI}(\mathrm{L})$ make up losses in the out cables, and $\mathrm{Dt}(\mathrm{H})$ and $\mathrm{Dt}(\mathrm{L})$ are both at zero voltage. (However, the ac QHR "standard" is defined between external stars $\mathrm{Y}$ and $\mathrm{Z}$; it therefore includes effects due to coaxial cables from the QHR device to those stars, as shown in the equivalent circuit model of Ref. [16].)

Differences in cable impedance between star $\mathrm{Z}$ to star $G$ and point $B$ to star $G$ contribute to the -3.2 parts in $10^{6}$ error in the 3TP mode from 4TP at dc. Only -2.4 of this -3.2 parts in $10^{6}$ error is due to differences in the out cable resistances. Furthermore, it would be difficult to correct for these differences to high accuracy: a $0.13 \mathrm{~m} \Omega$ variation of a connector contact resistance in the out cables would change the measured resistance ratio by 1 part in $10^{8}$. $3 \mathrm{TP}$ measurements are not adequate.

\section{$\Delta$ ac1/12.9WW1}

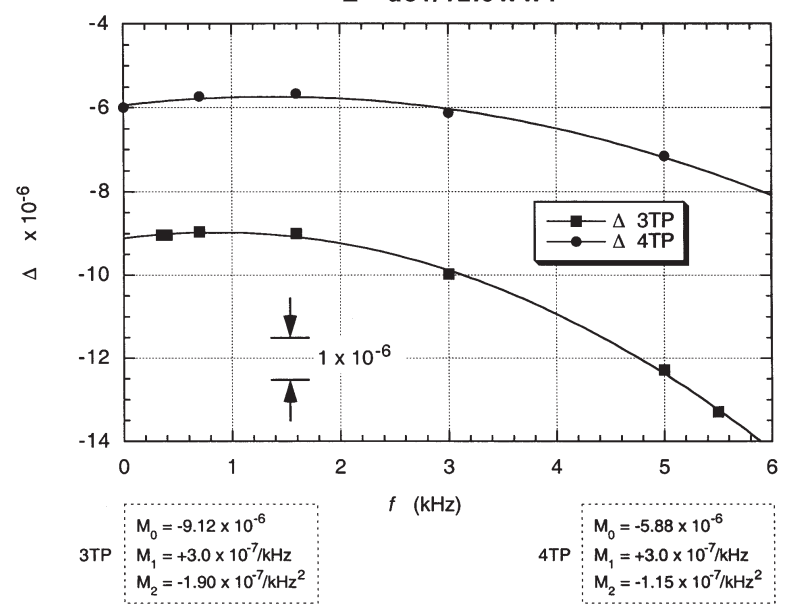

Fig. 16. Interchanged in-phase $\Delta$ deviations from the unity resistance ratio ac1/12.9WW1, obtained by averaging the differences between the "normal" and "exchanged" "raw data" of Figs. 13 and 14. The 4TP interchanged ac measurements converge within the \pm 1 part in $10^{7}$ uncertainty to the -6.0 parts in $10^{6} \mathrm{dc}$ value.

Note that the bridge linear frequency dependence is larger in magnitude than that of the resistance ratio: $(-5.0 \pm 0.1) \times 10^{-7} / \mathrm{kHz}$ compared with $(+3.0 \pm 0.1) \times$ $10^{-7} / \mathrm{kHz}$, and that the bridge quadratic frequency dependence is ten times larger: $(-12.07 \pm 0.10) \times$ $10^{7} / \mathrm{kHz}^{2}$ compared with $(-1.15 \pm 0.10) \times 10^{-7} / \mathrm{kHz}^{2}$. Bridge effects can dominate ac QHR $\alpha$ or $\alpha_{\mathrm{e}}$ frequencydependence measurements, and are unique to each bridge. It is thus crucial to perform interchanged measurements to obtain correct frequency dependences of resistance ratios. 
Fits to the data in Figs. $13-16$ are excellent, and are self-consistent for all three terms of every polynomial:

$$
\begin{array}{r}
2\left(M_{0}\right)_{\alpha_{0}}=\left(M_{0}\right)_{\alpha}+\left(M_{0}\right)_{\alpha_{\mathrm{e}}} \text { and } 2\left(M_{0}\right)_{\Delta}=\left(M_{0}\right)_{\alpha}-\left(M_{0}\right)_{\alpha_{\mathrm{e}}} \\
2\left(M_{1}\right)_{\alpha_{0}}=\left(M_{1}\right)_{\alpha}+\left(M_{1}\right)_{\alpha_{\mathrm{e}}} \text { and } 2\left(M_{1}\right)_{\Delta}=\left(M_{1}\right)_{\alpha}-\left(M_{1}\right)_{\alpha_{\mathrm{e}}}
\end{array}
$$

$2\left(M_{2}\right)_{\alpha_{0}}=\left(M_{2}\right)_{\alpha}+\left(M_{2}\right)_{\alpha_{\mathrm{e}}}$ and $2\left(M_{2}\right)_{\Delta}=\left(M_{2}\right)_{\alpha}-\left(M_{2}\right)_{\alpha_{\mathrm{e}}}$

The measurement uncertainties are \pm 1 part in $10^{7}$. Unfortunately the device characteristics prevented measurements to parts in $10^{8}$. That would have rigorously tested the quality of fits and convergence to the dc value.

\section{AC Quadrifilar Resistor Measurements}

Similar $20.0 \mu \mathrm{A}$ rms ac measurements and $29.1 \mu \mathrm{A}$ dc measurements comparing the $12906.4 \Omega$ quadrifilar resistor $12.9 \mathrm{QF} 1$ with the wire-wound reference resistor 12.9WW1 had been made nine months earlier. They are shown in Figs. 17-20. The ratio value was remeasured each day at $1592 \mathrm{~Hz}$ and adjusted to account for shifts of resistor 12.9QF1. Each datum point has a \pm 5 parts in $10^{8} 1 \sigma$ type A uncertainty. That could be reduced with more frequent measurements of the 12.9QF1 resistance shift, but this experiment was only intended as an initial mapping of bridge parameters.

"Raw data" 4TP interchanged resistance ratio deviation measurements $\Delta$ again converge to the $(42.0 \pm 0.1)$ parts in $10^{6} \mathrm{dc}$ value in Fig. 20. The "raw data" 3TP convergence error is about 4 parts in $10^{7}$. That is much smaller than the -3.2 parts in $10^{6}$ error in Fig. 16 for the ac $1 / 12.9 \mathrm{WW} 1$ ratio because out cable lengths (points $B$ to star $G$ ) between the two resistors are more closely-matched than the star Z to star G length to the ac QHR "standard".

All three bridge $\alpha_{0}$ polynomial terms of Fig. 19 are identical to those in Fig. 15 for both 3TP and 4TP measurements. Thus the bridge is very stable, and provides consistent results whether comparing the wirewound resistor with a QHR "standard" or with a quadrifilar resistor. Linear frequency dependence of the $12.9 \mathrm{QF} 1 / 12.9 \mathrm{WW} 1$ ratio is larger than that of the ac1/WW1 ratio: $(+4.9 \pm 0.1) \times 10^{-7} / \mathrm{kHz}$ compared with $\left.(+3.0 \pm 0.1) \times 10^{-7} / \mathrm{kHz}\right)$. The quadratic dependence is similar: $(-1.02 \pm 0.10) \times 10^{-7} / \mathrm{kHz}^{2}$ compared with $(-1.15 \pm 0.10) \times 10^{-7} / \mathrm{kHz}^{2}$.
Inadequate room airconditioning prevented direct comparisons of device ac1 with quadrifilar resistor 12.9QF1. Room temperatures rose above $24.5^{\circ} \mathrm{C}$ each day the magnet was used, causing $12.9 \mathrm{QF} 1$ to lose temperature control. Thus ac1/12.9QF1 intercomparisons await the move to a new laboratory. However, the ac1/12.9WW1 and $12.9 \mathrm{QF} 1 / 12.9 \mathrm{WW} 1$ intercomparisons imply that

$$
\begin{aligned}
& \mathrm{ac} 1 / 12.9 \mathrm{QF} 1=\left[1-(48.00 \pm 0.14) \times 10^{-6}\right] \\
& -\left[(1.90 \pm 0.14) \times 10^{-7} / \mathrm{kHz}\right] f \\
& -\left[\left(1.3 \pm 1.4 \times 10^{-8} / \mathrm{kHz}^{2}\right] f^{2} .\right.
\end{aligned}
$$

$\alpha \quad$ 12.9QF1/12.9WW1

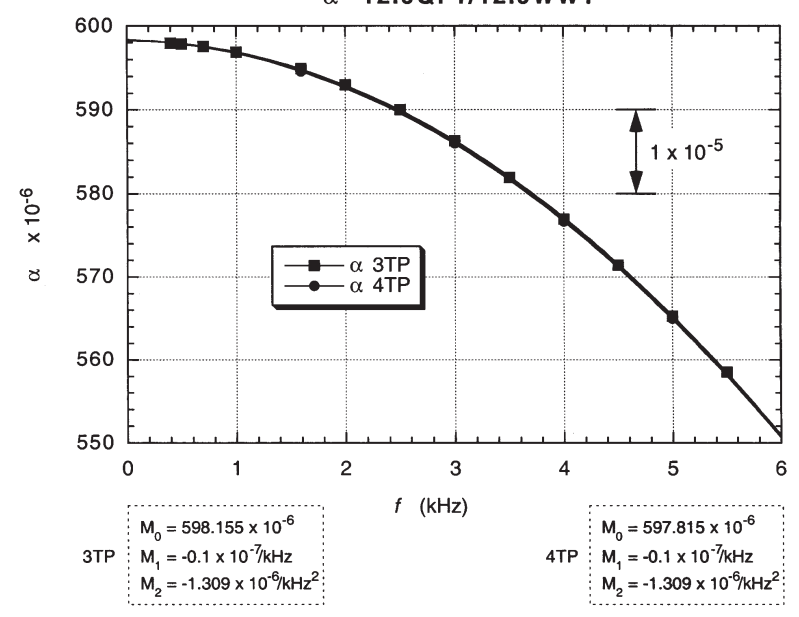

Fig. 17. "Raw data" 3TP and 4TP plots of $\alpha$ vs $f$ at $20.0 \mu \mathrm{A} \mathrm{rms,}$ $8.3 \mathrm{~T}$, and $1.51 \mathrm{~K}$ when $12.9 \mathrm{QF} 1$ and $12.9 \mathrm{WW} 1$ are in their "normal" bridge positions.

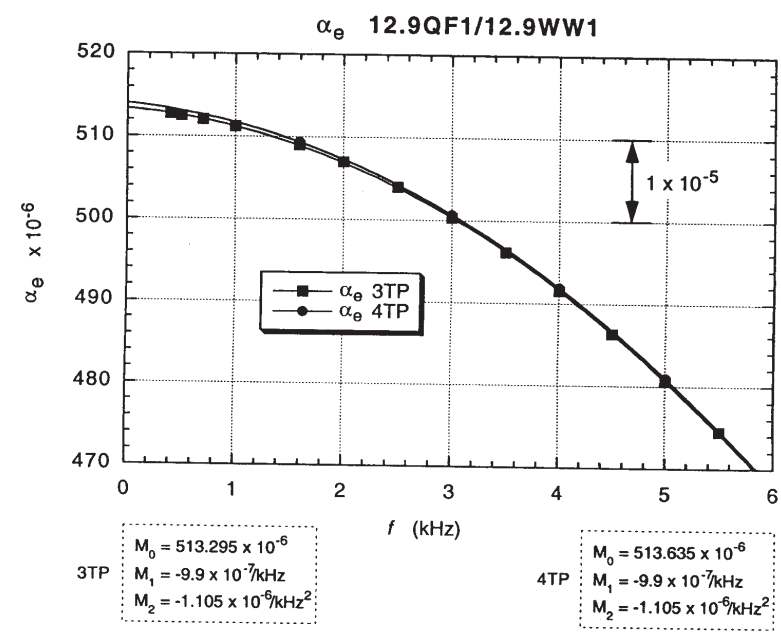

Fig. 18. "Raw data" 3TP and 4TP plots of $\alpha$ vs $f$ with 12.9QF1 and 12.9WW1 "exchanged" in the bridge. 


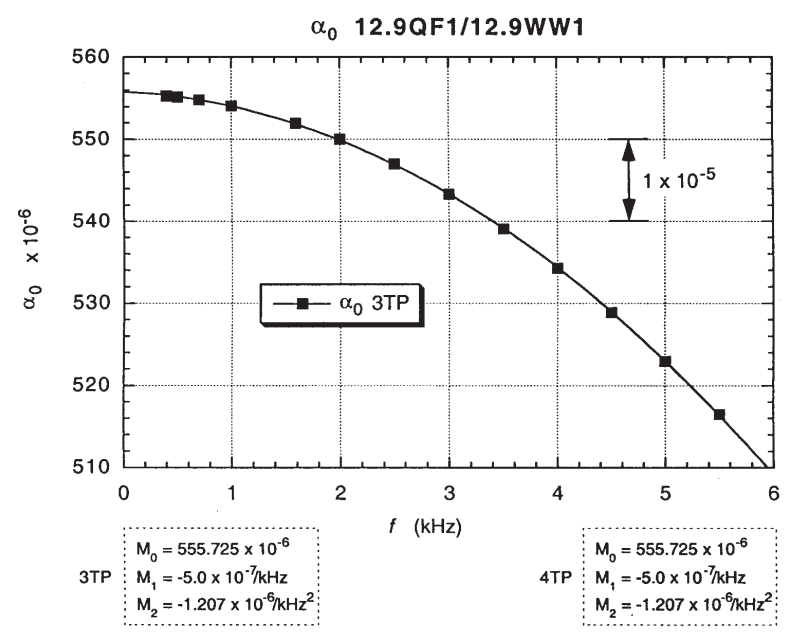

Fig. 19. AC bridge contributions $\alpha_{0}$ to the $\alpha$ and $\alpha_{\mathrm{e}}$ measurements, obtained by averaging the sums of "normal" and "exchanged" "raw data" of Figs. 17 and 18. These $\alpha_{0}$ vs $f$ results are the same for both $3 \mathrm{TP}$ and 4TP modes, and are identical to those of Fig. 15 for interchanged ac1/12.9WW1 measurements.

\section{$\triangle \quad 12.9 \mathrm{QF} 1 / 12.9 \mathrm{WW}^{1}$}

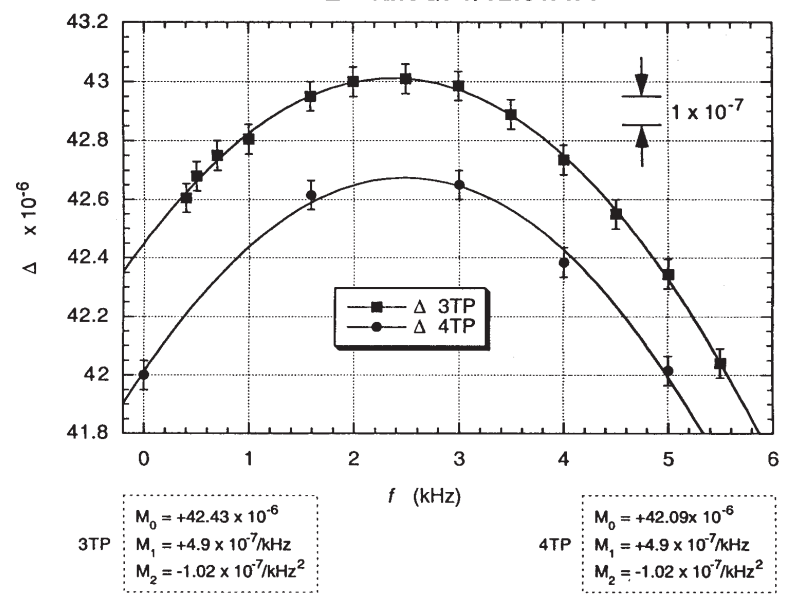

Fig. 20. Interchanged in-phase deviations $\Delta$ from the unity resistance ratio $12.9 \mathrm{QF} 1 / 12.9 \mathrm{WW} 1$, obtained by averaging differences between the "normal" and "exchanged" "raw data" of Figs. 17 and 18. The 4TP interchanged measurements converge within the \pm 1 part in $10^{7}$ uncertainty to the +42.0 parts in $10^{6} \mathrm{dc}$ value.

\section{Frequency Dependences}

Quadratic frequency dependences will be discussed in a future paper [24], but we see here that the bridge quadratic dependence was at least ten times larger than for any resistance ratio. Large linear frequency dependences appeared not only in measurements involving the ac QHR "standard," but also in the ac bridge, and in comparisons of two different types of resistors. Because of programmatic constraints, and since the device was of such poor quality, we did not pursue sources of the linear frequency dependence of the bridge, the ac1/12.9WW1 resistance ratio, or the 12.9QF1/12.9WW1 ratio. (Such as: moving the unchoked cable from probe 2 to other probe positions; adding chokes to the network; removing chokes; and verifying that the currents are indeed equal and opposite in the inner and outer conductors of every choke to test Schurr and Melcher's observations [25] that linear frequency dependences can be induced by improper choking and imperfect current equalization.)

\section{Conclusions}

DC QHR guideline properties and the dc and ac QHR values can be determined during one cooldown using single-series and quadruple-series connections outside the sample probe. The ac QHR values converged to the dc QHR value to within \pm 1 part in $10^{7}$ with a poor device when using external quadrupleseries connections and four-terminal-pair measurements. Convergence could be tested in the future to an order of magnitude smaller uncertainty with good devices. It was crucial to use 1:1 resistance ratios and make interchanged measurements to remove dominant ac bridge frequency-dependence effects.

\section{Acknowledgments}

We thank K. C. Lee at NIST for mounting the QHR device on one of our headers and pointing out the Corbino-like behavior of a potential contact, J. Q. Shields at NIST for designing most of the multifrequency transformer bridge, Y. Wang at NIST for resolving the correct signs of the ac resistance ratios (the transformer bridge had been originally designed to measure admittances rather than impedances), L. H. Lee at NIST for designing and constructing the wire-wound resistor, and constructing the auxiliary transformer and drive and out balances, R. F. Dziuba at NIST for helping trim the wire-wound resistance near the dc QHR value, J. Schurr of the PTB, Germany for private discussions about possible sources of linear frequency dependence, and G. N. Stenbakken and E. R. Williams at NIST, and A. D. Inglis and B. M. Wood at NRC, Canada for their discussions, suggestions, and comments. 


\section{References}

[1] J. Melcher, P. Warnecke, and R. Hanke, Comparison of Precision AC and DC Measurements with the Quantized Hall Resistance, IEEE Trans. Instrum. Meas. 42, 292-294 (1993).

[2] F. Delahaye, Accurate AC Measurements of the Quantized Hall Resistance from $1 \mathrm{~Hz}$ to $1.6 \mathrm{kHz}$, Metrologia 31, 367-373 (1995).

[3] A. Hartland, B. P. Kibble, P. J. Rodgers, and J. Bohacek, AC Measurements of the Quantized Hall Resistance, IEEE Trans. Instrum. Meas. 44, 245-248 (1995).

[4] B. M. Wood, A. D. Inglis, and M. Cote, Evaluation of the AC Quantized Hall Resistance, IEEE Trans. Instrum. Meas. 46, 269-272 (1997).

[5] J. Bohacek, P. Svoboda, and P. Vasek, AC QHE-Based Calibration of Resistance Standards, IEEE Trans. Instrum. Meas. 46, 273-275 (1997).

[6] B. M. Wood, A. D. Inglis, M. Cote, and R. B. Young, Improved AC Quantized Hall Measurements, IEEE Trans. Instrum. Meas. 48, 305-308 (1999).

[7] S. W. Chua, A. Hartland, and B. P. Kibble, Measurement of the AC Quantized Hall Resistance, IEEE Trans. Instrum. Meas. 48, 309-313 (1999).

[8] F. Delahaye, B. P. Kibble, and A. Zarka, Controlling AC Loses in Quantum Hall Effect Devices, Metrologia 37, 659-670 (2000).

[9] J. Schurr, J. Melcher, A. von Campenhausen, G. Hein, F. -J. Ahlers, and K. Pierz, AC Behaviour and Loss Phenomena in Quantum Hall Samples, Metrologia 39, 2-12 (2002).

[10] J. Schurr, J. Melcher, A. von Campenhausen, and K. Pierz, Adjusting the Losses in an AC Quantum Hall Sample, Metrologia 39, 13-19 (2002).

[11] K. von Klitzing, G. Dorda, and M. Pepper, New Method for High-Accuracy Determination of the Fine-Structure Constant Based on Quantized Hall Resistance, Phys. Rev. Lett. 45, 494497 (1980).

[12] The Quantum Hall Effect, R. E. Prange and S. M. Girvin, eds., Springer-Verlag, New York (1987) pp. 1-419.

[13] The Integral and Fractional Quantum Hall Effects, C. T. Van Degrift, M. E. Cage, and S. M. Girvin, eds., American Association of Physics Teachers, College Park, Maryland (1991) pp. 1-116.

[14] F. Delahaye, Technical Guidelines for Reliable Measurements of the Quantized Hall Resistance, Metrologia 26, 237-240 (1989); F. Delahaye and B. Jeckelmann, Revised Technical Guidelines for Reliable DC Measurements of the Quantized Hall Resistance, Metrologia 40, 217-223 (2003).

[15] F. Delahaye, Series and Parallel Connection of Multiterminal Quantum Hall Effect Devices, J. Appl. Phys. 73, 7915-7920 (1993).

[16] M. E. Cage, A. Jeffery, and J. Matthews, Equivalent Electrical Circuit Representations of AC Quantized Hall Resistance Standards, J. Res. Natl. Inst. Stand. Technol. 104 (6), 529-556 (1999).

[17] R. D. Cutkosky, Four-Terminal-Pair Networks as Precision Admittance and Impedance Standards, Commun. Electron. 70, 19-22 (1964).

[18] R. D. Cutkosky, Techniques for Comparing Four-Terminal-Pair Admittance Standards, J. Res. Natl. Bur. Stand. (U.S.) 74C, 6378 (1970).

[19] A. Jeffery, R. E. Elmquist, J. Q. Shields, L. H. Lee, M. E. Cage, S. H. Shields, and R. F. Dziuba, Determination of the von
Klitzing Constant and the Fine-Structure Constant Through a Comparison of the Quantized Hall Resistance and the Ohm Derived from the NIST Calculable Capacitor, Metrologia 35, 83-96 (1998).

[20] D. L. H. Gibbings, A Design for Resistors of Calculable a.c./d.c. Resistance Ratio, Proc. IEE 110, 335-347 (1963).

[21] G. M. Reedtz and M. E. Cage, An Automated Potentiometric System for Precision Measurement of the Quantized Hall Resistance, J. Res. Natl. Bureau Stand. (U.S.). 92 (5), 303-310 (1987).

[22] D. N. Homan, Applications of Coaxial Chokes to AC Bridge Circuits, J. Res. Natl. Bur. Stand. (U.S.) 72C, 161-165 (1968).

[23] A. Jeffery, J. Q. Shields, and L. H. Lee, An Easy-To-Use Combination Four-Terminal-Pair/Two-Terminal-Pair AC Transformer Bridge, J. Res. Natl. Inst. Stand. Technol. 103 (2), 163-166 (1998).

[24] M. E. Cage, Quadratic Frequency Dependence of an AC QHR Device, to be published.

[25] J. Schurr and J. Melcher, CPEM2002, Ottawa, Canada, June 16-21 (2002) and private communication.

About the authors: M. E. Cage is a physicist and S. H. Shields a technician in what was the Electricity Division and is now the Quantum Electrical Metrology Division of the Electronics and Electrical Engineering Laboratory of the National Institute of Standards and Technology. A. Jeffery was a physicist in the Electricity Division and has now graduated from seminary school. The National Institute of Standards and Technology is an agency of the Technology Administration, U.S. Department of Commerce. 